\title{
Facile Fabrication of Biomimetic Water Pinning Microstructures on Polyethylene Surfaces with Robust Superhydrophobic Wetting State
}

\author{
Yuzhu $\mathrm{Li}^{1,2}$, Hang Gao ${ }^{1,2^{*}}$, Yunhai $\mathrm{Ma}^{3}$, Donghui Chen ${ }^{3}$ \\ 1. School of Mechatronics Engineering, Shenyang Aerospace University, Shenyang 110136, China \\ 2. Key Laboratory of Fundamental Science for National Defense of Aeronautical Digital Manufacturing Process, \\ Shenyang 110136, China \\ 3. Key Laboratory for Bionic Engineering, Ministry of Education, Jilin University, Changchun 130022, China
}

\begin{abstract}
Applying hot-embossing technology, a simple and cost-effective method for the fabrication of microstructured High Density Polyethylene (HDPE) surfaces with a robust superhydrophobic wetting state is proposed. Micro-meshes and micro-grooves in the flexible template are filled by the PE melt in the hot embossing process. Subsequently, a two-stage microstructure on the PE film surface is formed. This PE film exhibits a contact angle of $151.8^{\circ} \pm 2^{\circ}$ and roll-off angle of $>90^{\circ}$ when a $5 \mu \mathrm{L}$ water droplet is dropped on its surface. Water pinning ability on the surface is figured out and roll-off angles are as a quadratic function of specified water droplet volume. Specifically, a $356 \mu \mathrm{N}$ water pinng force appears on the HDPE film due to the solid-vapor composite interface on its surface. Meanwhile, self-cleaning and immersion tests reveal that the HDPE surface with micro-pillars exhibit robust Cassie impregnating wetting state against external pressure. The proposed method for facial fabrication of microstructured surfaces is an appropriate candidate for the development of droplet manipulation and functional biomimetic polymer surfaces.
\end{abstract}

Keywords: water pinning, hot-embossing, Cassie impregnating, self-cleaning, microstructure

Copyright $(\odot)$ The author(s) 2020 .

\section{Introduction}

Through uninterrupted evolution and natural selection, natural creatures have evolved various well-designed structures with excellent features for living in harsh environments ${ }^{[1,2]}$. It stands to reason that these well-designed structures are ideal innovation sources for new materials, structures, methods, and tools. Examples include self-cleaning lotus leaf ${ }^{[3]}$, structural color butterfly wing ${ }^{[4]}$, robust superhydrophobic water strider $1 \mathrm{lg}^{[5]}$, high adhesive gecko foot ${ }^{[6]}$, superhydrophobic and antireflection poplar leaf ${ }^{[7]}$ and fog harvesting cicada wing ${ }^{[8]}$. Micro/nanostructured surfaces with extreme wettability have attracted significant attention due to their broad technological applications ranging from water harvesting, anticorrosion, liquid transport in microfluidics, anti-icing, self-cleaning and oil//water separation ${ }^{[9-12]}$.

As one of the typical biological prototypes, rose petal is well-known for its strong water pinning property where a water droplet does not roll off the petal surface even when it is turned upside down ${ }^{[13]}$. The rose petal surface exhibits superhydrophobic wetting state yet strong water droplet adhesion, namely, petal effect. Micro/nanostructured surfaces with petal effect have wide applications in the fields of fog collection in arid regions, anti-drip films in agricultural countries, liquid control in microfluidics, and coffee-ring effect manipulation in printing and coating technologies ${ }^{[14-17]}$. Undoubtedly, there will definitely be a huge market potential to commercialize the idea of preparing micro/nanostructure surfaces with petal effect in mass production.

Polymer material is one of the most widely used materials for its advantages of relatively low price, excellent compatibility, easy handling and processing with special features. Creating micro/nanostructures with special properties on polymer surfaces has attracted significant attention due to their potential applications in fundamental research and biomedical industry ${ }^{[18-22]}$. Hot embossing is a common method to create micro-

\footnotetext{
*Corresponding author: Hang Gao
}

E-mail: saugaohang@163.com 
nanostructures on a thermoplastic polymer surface, as thermoplastics have the ability to become moldable above a certain glass transition temperature and return to their solid state by cooling ${ }^{[23]}$. However, creating water pinning microstructures on polymer surfaces still suffers from limitations that restrict its wide application. First, fabricating micro- nanostructures on polymer surfaces by hot embossing involves creating a template, typically on a hard metallic surface and using this template to reproduce its reverse pattern on a polymeric substrate. A variety of technologies, such as laser ablation, anodization of aluminum oxide, micro-milling, photo-lithography, and electroforming have been used for the preparation of the template with micronanostructures ${ }^{[2-28]}$. The template preparation methods are mainly involved in a complex process or expensive device. The method is faced with problems like harsh conditions, high cost, environmental unfriendliness, and a narrow selection of materials. Moreover, spatial patterning precision and of the techniques template are unsuitable for mass production.

To pursue facile preparation of the water pinning microstructures on High Density Polyethylene (HDPE) surface, an approach that combines hot-embossing and flexible plain-woven mesh is proposed in our work. As a molding method, hot-embossing is typically used in the highly efficient and low-cost fabrication of micronanostructured polymer surfaces ${ }^{[29-31]}$. Using the proposed approach, HDPE films with orderly arranged tapered microstructures are fabricated, which exhibit water pinning and self-cleaning properties.

\section{Experiment}

\subsection{Materials and sample preparation}

HDPE (grade FY1002, Fanya Polyester Co. Ltd., China) was used as received. A hot-embossing machine (QBL-350, Wuxi No.1 Rubber \& Plastics Mechanical Co. Ltd., China) coupled with a steel mold was used to fabricate rectangular HDPE surfaces with nominal dimensions of $240 \times 240 \times 1.5 \mathrm{~mm}^{3}$. A flexible plain-woven mesh (\#800-mesh) with a thickness of $70 \mu \mathrm{m}$ was used as a template to fabricate microstructures on the surface of the HDPE film.

The overall process for preparing the water pinning microstructures on HDPE surfaces is shown in Fig. 1.
First, the template (Fig. 1a) was ultrasonically cleaned with deionized water, followed by drying in a vacuum oven at $80{ }^{\circ} \mathrm{C}$. Then the template was placed on the fixed platform of the hot-embossing machine (Fig. 1b) and dried HDPE pellets were placed over the template (Fig. 1c). Finally, the HDPE pellets were compressed into the template with nominal dimensions of $120 \times 120$ $\times 1.5 \mathrm{~mm}^{3}$ by the hot-embossing machine at the temperature and force of $170{ }^{\circ} \mathrm{C}$ and $15 \mathrm{MPa}$, respectively (Fig. 1d). Importantly, the HDPE pellets were placed over the template and pressed by the moving platform for compression duration of $300 \mathrm{~s}$ at the controlled compression temperature $\left(150{ }^{\circ} \mathrm{C}\right)$ above the glass transition temperature $\left(T_{\mathrm{m}}\right)$ of $\operatorname{HDPE}\left(130{ }^{\circ} \mathrm{C}\right)$ that allows the softened viscous HDPE to completely fill the micro-meshes and micro-grooves in the template. After cooling and demolding, dense and orderly tampered microstructures were formed on the surface of the HDPE film (Fig. 1e). For comparison, bare HDPE films were molded under the same molding parameters without the plain-woven mesh as the template.

\subsection{Characterization}

The surface topographies of the plain-woven mesh and HDPE films were examined by Scanning Electron Microscopy (SEM; XL-30-ESEM, FEI Co., Nether-lands) at an accelerating voltage of $10 \mathrm{kV}$. Prior to the SEM observation, the template and HDPE films were coated with gold by ion sputtering. The Contact Angles (CAs) and Roll-off Angles (RAs) of the HDPE films were measured using an automatic contact-angle testing apparatus (OCA 20, Dataphysics, Germany) to describe the static and dynamic wettability, respectively. Both CA and RA measurements were conducted at five different locations on the film to obtain the average values. Photographs and videos of the self-cleaning and immersion behaviors were captured using an SLR camera equipped with an EF $18 \mathrm{~mm}-135 \mathrm{~mm}$ lens (Canon 6d, Japan).

\section{Results and discussion}

\subsection{Microstructures of template and HDPE film}

The morphology of plain-woven mesh template and microstructures on HDPE film captured by the SEM are shown in Fig. 2. As can be seen in Fig. 2a, the template is 


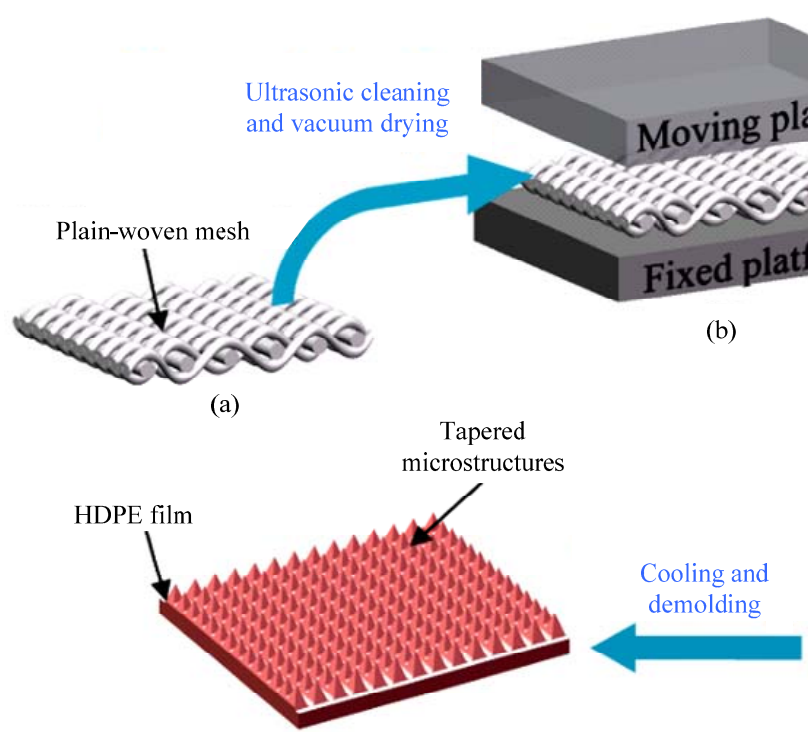

(e)

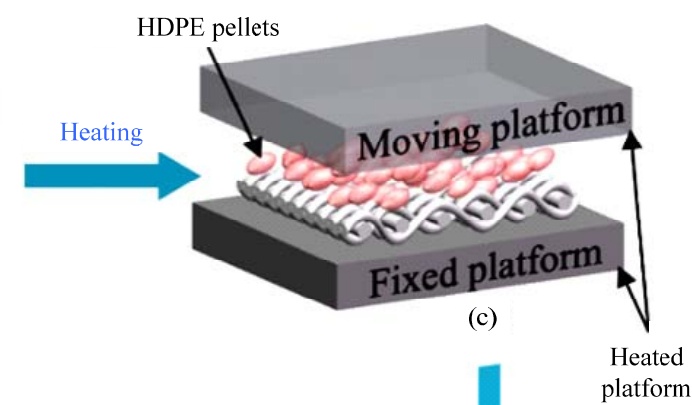

(c)

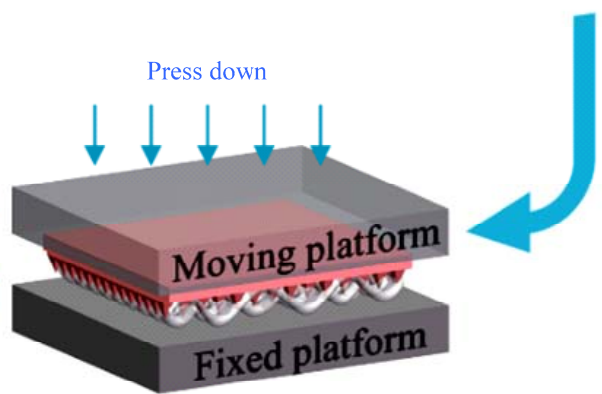

(d)

Fig. 1 Schematics of preparing processes for microstructured HDPE films.

composed of a set of weft wires and warp wires. The wires interlace with one another in the template and the warp wires are larger in diameter than the weft wires, thus forming micro-meshes and micro-grooves. The HDPE melt fill the micro-meshes, overflow into the micro-grooves to form the micro-pillars and micro-edges (Fig. 2b), respectively. The HDPE material has good strength and ductility, and the mesh is flexible. So, the micropillars can be detached from the template with slight deformation and without residue during demolding. Therefore, a two-stage microstructure, micro-pillars and micro-edges, orderly and densely arranged micro-pillars appear on the HDPE surface (Figs. 2b and 2c).

\subsection{Wetting behavior on HDPE film surface}

The macroscopic wetting states for $8 \mu \mathrm{L}-12 \mu \mathrm{L}$ water droplet on the bare and microstructured HDPE films are shown in Figs. $3 a$ and $3 c$, respectively. It is obvious that the bare HDPE film exhibits a hydrophilic wetting state (Fig. 3a) and the microstructured HDPE film exhibits a hydrophobic wetting state (Fig. 3c), Quantitatively, the $5 \mu \mathrm{L}$ water droplet dripping on bare HDPE film exhibits a CA of $64.8^{\circ}$ (Fig. 3b). Interestingly, the HDPE film with the two-stage microstructures exhibits a CA of $151.8^{\circ} \pm 2^{\circ}$ and $\mathrm{RA}$ of $>90^{\circ}$ when the water
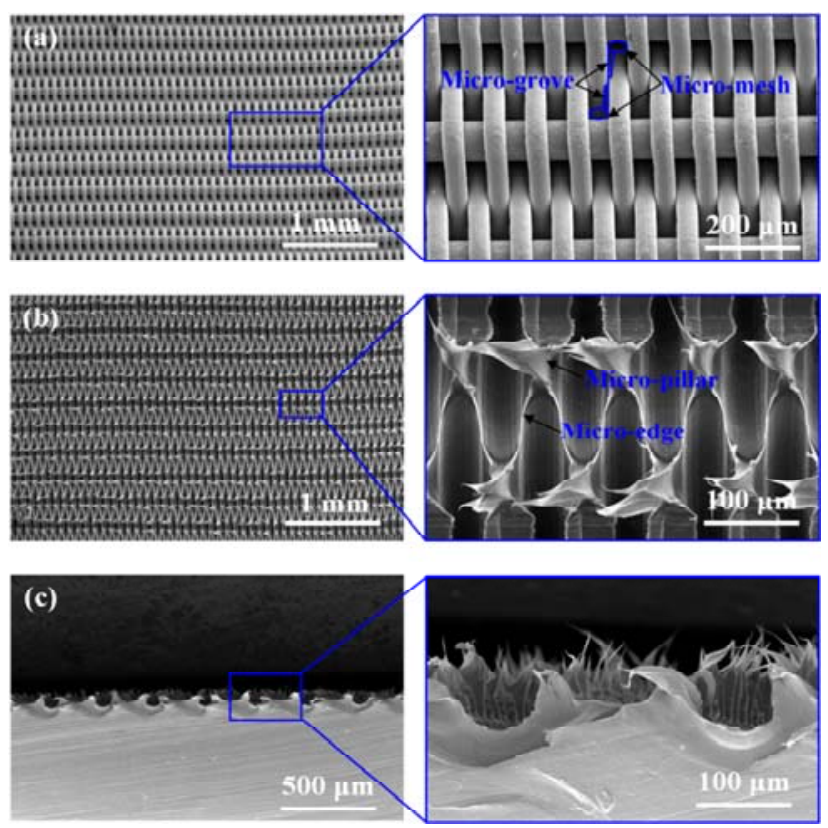

Fig. 2 Morphologies of the template (a) and microstructures on HDPE film surface $(b, c)$.

droplets with the same volume are dropped on its surface (Fig. 3d). Undoubtedly, the aforementioned two-stage microstructure on the HDPE film surface is closely correlated with its surface wetting behavior. A typical petal effect, high static water CA yet strong water droplet pinning force $\left(F_{\mathrm{p}}\right)$, appears on the microstructured HDPE surface for a $5 \mu \mathrm{L}$ water droplet. 
In order to figure out water pinning ability on the surface of the microstructured HDPE film, more $5 \mu \mathrm{L}$ water droplets are added to the original $5 \mu \mathrm{L}$ droplet. With the growth of droplets in size and the increase of the tilted angle $\left(\leq 90^{\circ}\right)$, the pinned droplets finally roll off the microstructured HDPE film under the action of gravity. The RAs of water droplets of different volumes are measured at five different locations to calculate the average value and the results are shown in Fig. 4. The relationship curve between RA and droplet volume can be divided into two segments. When the volume of the water droplet is less than or equal to $25 \mu \mathrm{L}$, the droplets are still unable to overcome the $F_{\mathrm{p}}$ to roll off the microstructured surface even if the film tilts to the maximum angle $\left(90^{\circ}\right)$. However, when the droplet volume increases to $30 \mu \mathrm{L}$, it rolls off the microstructured HDPE film at the tilted angle of $81^{\circ}$. RAs of the film are subsequently reducing with the increase of droplet volume. Through fitting the data between $35 \mu \mathrm{L}$ and $60 \mu \mathrm{L}$, we can obtain a polynomial equation as follows:

$$
y=160.2-3.3 x+0.02 x^{2}, 25 \leq x \leq 60,
$$

where $x$ is the droplet volume $(\mu \mathrm{L})$ and $y$ is the tilted angle of the HDPE film. The water droplets with different volumes can be collected on the microstructured films tilted at different angles, which can be used to collect and transfer water droplet quantitatively.

To qualitatively evaluate the self-cleaning ability on the microstructured HDPE film, silica sands as contaminants are spread on the $30^{\circ}$ tilted film, as shown in Fig. 5a. Then, water droplets are dropped on the film (Fig. 5b). The droplets roll off the film spontaneously and the silica sands could be washed by the droplet easily (Fig. $5 \mathrm{c}$ ), indicating the self-cleaning ability appears on the microstructured HDPE surface with petal effect. The whole self-cleaning test is recorded by a camera and is displayed supplementary video $\mathrm{S} 1$.

Furthermore, the stability of the superhydrophobic state against the external pressure on the microstructured HDPE film is investigated by an immersion test. Water used in the immersion test is dyed blue to aid visualization by blue ink $(0.1 \mathrm{wt} \%)$, as shown in Fig. $6 \mathrm{a}$.

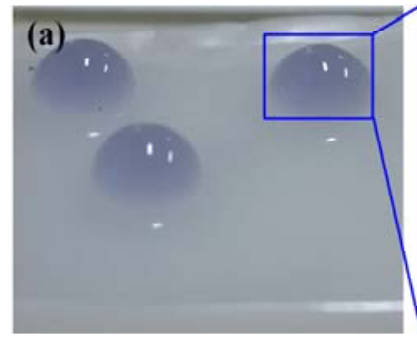

(b)
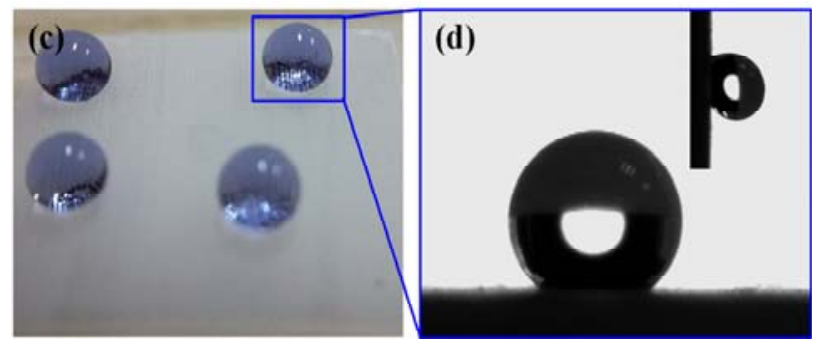

Fig. 3 (a) Wetting state and (b) CA on bare HDPE film; (c) wetting state and (d) CA on microstructured HDPE film.

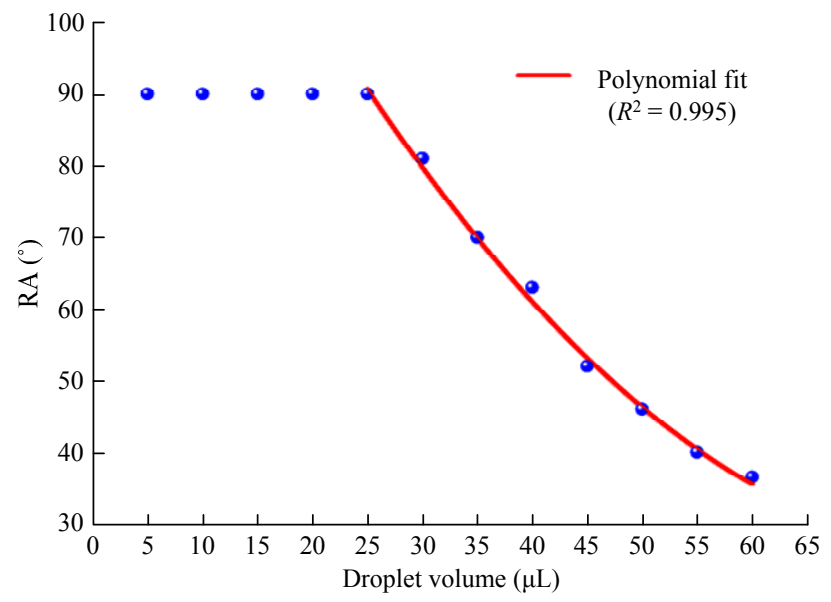

Fig. 4 Relationship between RA and droplet volume.

The HDPE film is not immediately immersed in the blue ink dyed water due to the superhydrophobic interaction between the blue ink dyed water and the microstructured surface, as indicated by the convex contact interface (Fig. 6b). Finally, the microstructured HDPE film is immersed in the blue water under a sufficient vertical force (Fig. 6c). Obviously, the film remains fully spotless with no trace of contamination after holding $5 \mathrm{~s}$ and taking it off from the dyed water (Fig. 6d). The whole immersion test is recorded by a camera and is displayed by supplementary video S2. Consequently, the microstructured HDPE film exhibits robust superhydrophobic state and water repellent property. 

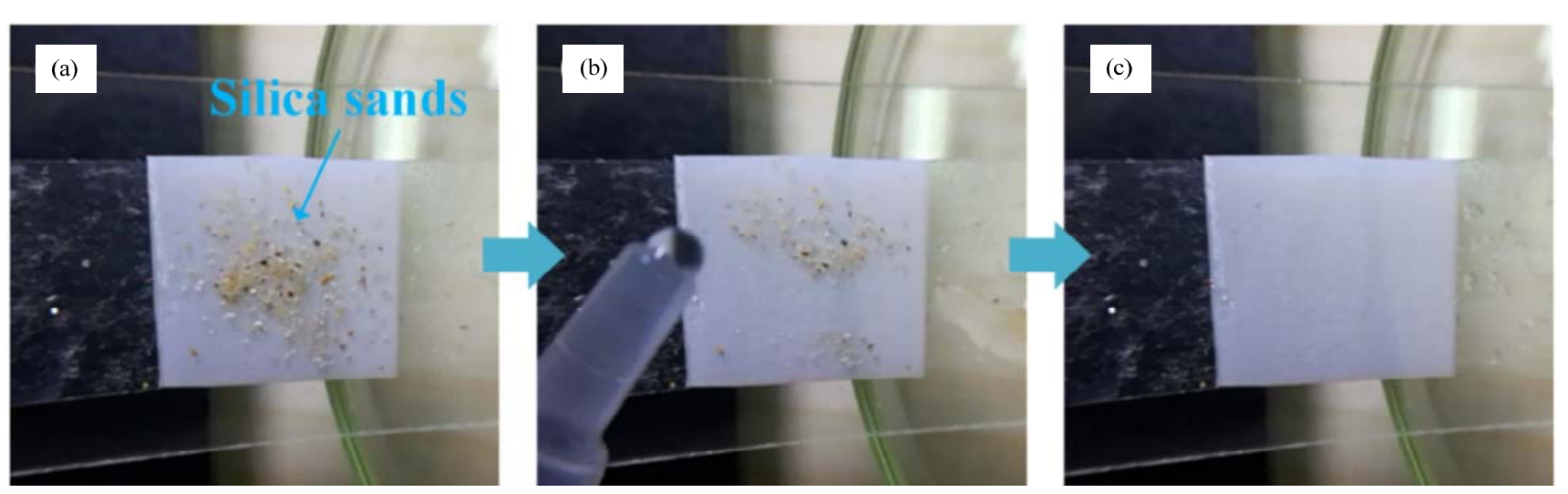

Fig. 5 Images of $30^{\circ}$ tilted HDPE film contaminated by silica sands (a) before, (b) during and (c) after droplets washing.
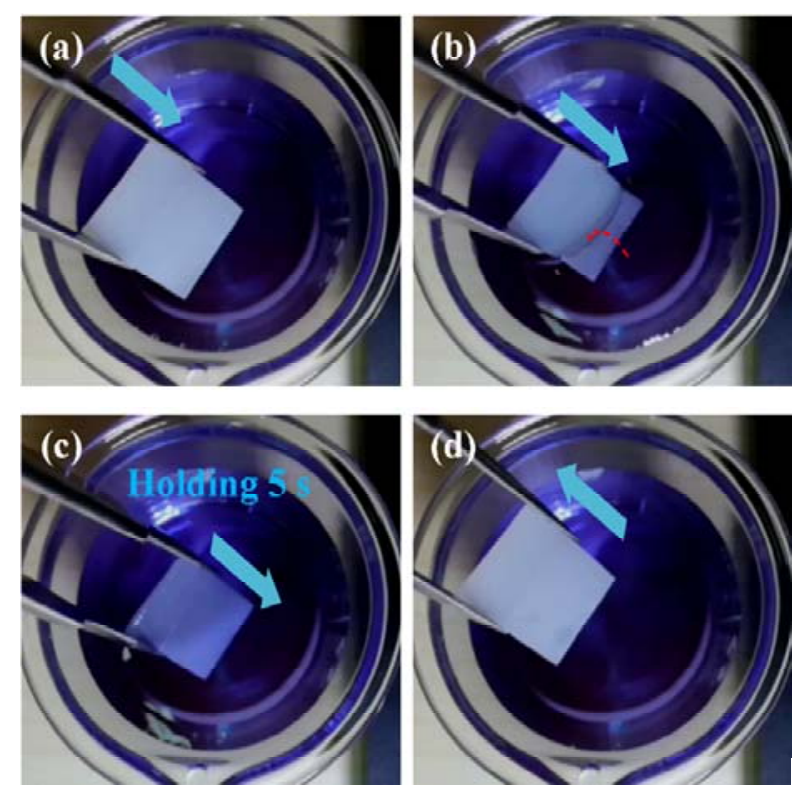

Fig. 6 Typical images of microstructured HDPE film (a) before, $(b, c)$ during and (c) after drowning in blue ink dyed water.

\subsection{Mechanism of wetting behavior and relevant water pinning force}

The underlying mechanisms for the wetting behaviors on the bare and microstructured HDPE film surfaces are depicted in Fig. 7. The bare HDPE film exhibits an obvious hydrophilic wetting state (Fig. 3a) which can be attributed to the interaction between surface tensions of the solid-liquid $\left(\gamma_{\mathrm{SL}}\right)$, liquid-vapor $\left(\gamma_{\mathrm{LV}}\right)$ and solid-vapor $\left(\gamma_{\mathrm{SV}}\right)$. According to Young's equation, $\gamma_{\mathrm{SL}}$ and $\gamma_{\mathrm{LV}}$ attempt to make the water droplet to shrink, whereas $\gamma_{\mathrm{SV}}$ attempts to spread out the droplet $^{[32,33]}$. When the surface tensions reach equilibrium, the angle between the solid-liquid interface and the liquid-vapor interface is the $\mathrm{CA}(\theta)$ of bare HDPE

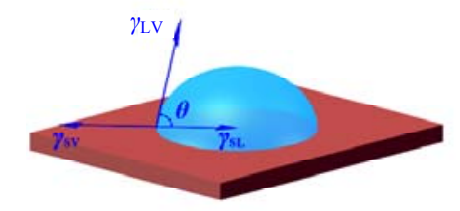

(a)

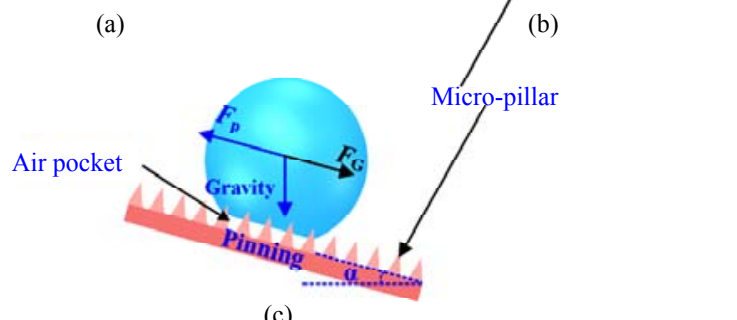

(c)
Fig. 7 Mechanisms for (a) hydrophilic and (b, c) Cassie impregnating states on HDPE film surface.

film (Fig. 7a). Due to the hydrophilic nature of HDPE, it is energetically profitable for a droplet to spread on the surface rather than to shrink. So the bare HDPE film exhibits a low CA. As a microstructured film, orderly and densely arranged micro-pillars appear on the HDPE surface. The upper portion of the micro-pillars is easily impregnated by water, and the air is trapped in the gaps between the micro-pillars to form a stable air pocket that acts as an effective water barrier ${ }^{[34]}$. So the bottom portion of the micro-pillars still remains dry (Figs. $7 b$ and 7c). A solid-vapor composite interface is formed on the microstructured HDPE surface, resulting in a high CA (Fig. 3b) and an intermediate state between the Wenzel and Cassie-Baxter states called "Cassie impregnating state" exhibit the microstructured HDPE surface $^{[13,35]}$.

The pinning force between a droplet and a microstructured surface is first recommended by 
Fumidge in 1962 and subsequently applied by other research groups ${ }^{[36-38]}$. The maximum size of a droplet that can fix on a titled surface can be calculated by balancing the capillary force caused by micronanostructures with the gravitational force. As can be seen from Fig. 7c, water droplet with a moderate volume can be fixed on the tilted HDPE film surface with the micro-pillars. The droplet rolls off the surface when its volume reaches a specified value because its gravity component $\left(F_{\mathrm{G}}\right)$ overcomes the water pinning force $\left(F_{\mathrm{p}}\right)^{[39]}$. Specifically, the pinning force to the water droplet on the microstructured HDPE film surface is calculated using the following equation ${ }^{[40]}$ :

$$
F_{\mathrm{p}}=F_{\mathrm{G}}=m g \sin \alpha,
$$

where $m$ and $g$ represent the mass of the water droplet and the acceleration of gravity, respectively. $\alpha$ shows the tilting angle at the onset of droplets rolling off the microstructured HDPE surface. Substituting the RA and volume of a water droplet in Fig. 3 to Eq. (2), $F_{\mathrm{p}}$ of the microstructured HDPE film is calculated to be $356 \mu \mathrm{N}$, which is better than the water pinning force of the natural rose petal $(63.8 \mu \mathrm{N})^{[37]}$ and many advanced synthetic structures $(15 \mu \mathrm{N}-230 \mu \mathrm{N})$ reported by other research teams ${ }^{[41-45]}$.

Furthermore, the water droplet adheres to the upper portion of the micro-pillars, so $F_{\mathrm{p}}$ is closely related to the droplet contact diameter. Fig. 8 shows the shape and contact diameter of water droplets $(5 \mu \mathrm{L}, 10 \mu \mathrm{L}, 15 \mu \mathrm{L})$ on the microstructured HDPE surface. It is clear that the contact diameter increases with the increasing droplet volume, because of the larger liquid-solid contact area and thus higher adhesion of the droplet to the film surface. Interestingly, the droplets with different volume still maintain their spherical shape and high $\mathrm{CA}$ which indicates from a side that the microstructured HDPE film has a stability wetting state against the external pressure.

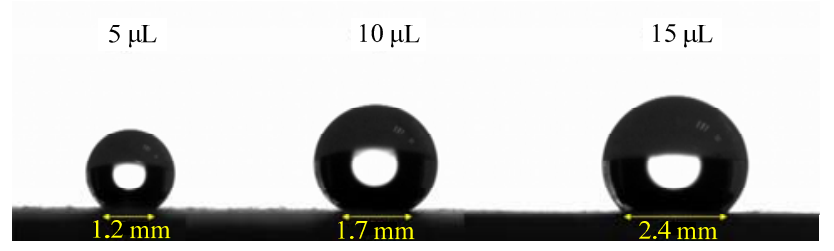

Fig. 8 Shape and contact diameter of water droplets on the microstructured surface of HDPE film.

\section{Conclusion}

In conclusion, superhydrophobic microstructured HDPE films with high water pinning force have been achieved through hot-embossing. Low cost flexible plain-woven mesh is favorable for the fabrication of the two-stage microstructured HDPE film which exhibits a CA of $151.8^{\circ} \pm 2^{\circ}$ and RA of $>90^{\circ}$. High static water CA yet strong water droplet pinning force appears on the microstructured HDPE surface for a $5 \mu \mathrm{L}$ water droplet. Specifically, the roll-off angles on such HDPE surface are as a quadratic function of specified water droplet volume. Self-cleaning and immersion tests reveal that the HDPE surface with micro-pillars exhibit robust Cassie impregnating wetting state against external pressure, which is attributed to both surface tension and trapped air pocket. The upper portion of the micro-pillars is impregnated by a water droplet and so a $356 \mu \mathrm{N}$ water pinng force appears on the HDPE film. Applying a technique combing hot-embossing and flexible plain-woven mesh, a straightforward and facile route is provided for mimicking bio-inspired microstructures with sufficient water pinning force.

\section{Acknowledgment}

This work was supported by the Nature Science Foundation of China (Grant No. 51475205), Research Foundation for Introduced Talents of Shenyang Aerospace University (502/120419030 and 502/120419031).

* All supplementary materials are available at https://doi.org/10.1007/s42235-020-0052-1.

Open Access This article is licensed under a Creative Commons Attribution 4.0 International License, which permits use, sharing, adaptation, distribution and reproduction in any medium or format, as long as you give appropriate credit to the original author(s) and the source, provide a link to the Creative Commons licence, and indicate if changes were made.

The images or other third party material in this article are included in the article's Creative Commons licence, unless indicated otherwise in a credit line to the material. If material is not included in the article's Creative Commons licence and your intended use is not permitted by statutory regulation or exceeds the 
permitted use, you will need to obtain permission directly from the copyright holder.

To view a copy of this licence, visit http://creativecommons.org/licenses/by/4.0/.

\section{References}

[1] Koch, K, Barthlott W. Superhydrophobic and superhydrophilic plant surfaces: An inspiration for biomimetic materials. Philosophical Transactions of the Royal Society of London Series A, 2009, 367, 1487-1509.

[2] Liu M J, Wang S T, Lei J. Nature-inspired superwettability systems. Nature Reviews Materials, 2017, 2, 17036.

[3] Ensikat H J, Ditsche-Kuru P, Neinhuis C, Barthlott W. Superhydrophobicity in perfection: The outstanding properties of the lotus leaf. Beilstein Journal of Nanotechnology, 2011, 2, 152-161.

[4] Poladian L, Wickham S, Lee K, Large M C. Iridescence from photonic crystals and its suppression in butterfly scales. Journal of the Royal Society Interface, 2009, 6, 233-242.

[5] Bai F, Wu J T, Gong G M, Guo L. Biomimetic "water strider leg" with highly refined nanogroove structure and remarkable water-repellent performance. ACS Applied Materials \& Interfaces, 2014, 6, 16237-16242.

[6] Sitti M, Fearing R S. Synthetic gecko foot-hair micro/nano-structures as dry adhesives. Journal of Adhesion Science and Technology, 2003, 18, 1055-1073.

[7] Ye C Q, Li M Z, Hu J P, Cheng Q F, Jiang L, Song Y L. Highly reflective superhydrophobic white coating inspired by poplar leaf hairs toward an effective "cool roof". Energy \& Environmental Science, 2011, 4, 3364-3367.

[8] Xie H, Huang H X, Mi H Y. Gradient wetting state for droplet transportation and efficient fog harvest on nanopillared cicada wing surface. Materials Letters, 2018, 221, 123-127.

[9] Wang S T, Liu K S, Yao X, Lei J. Bioinspired surfaces with superwettability: New insight on theory, design, and applications. Chemical Reviews, 2015, 115, 8230-8293.

[10] Zhang S N, Huang J Y, Chen Z, Lai Y K. Bioinspired special wettability surfaces: From fundamental research to water harvesting applications. Small, 2017, 13, 1602992.

[11] Xie Y H, Chen H F, Shen Y Z, Tao J, Jin M M, Wu Y, Hou W Q. Rational fabrication of superhydrophobic nanocone surface for dynamic water repellency and anti-icing potential. Journal of Bionic Engineering, 2019, 16, 27-37.

[12] Liu W L, Chen H F, Shen Y Z, Wu Z W. Facilely fabricating superhydrophobic resin-based coatings with lower water freezing temperature and ice adhesion for anti-icing application. Journal of Bionic Engineering, 2019, 16, 794-805.

[13] Feng L, Zhang Y N, Xi J M, Zhu Y, Wang N, Xia F, Jiang L. Petal effect: A superhydrophobic state with high adhesive force. Langmuir, 2008, 24, 4114-4119.

[14] Tian X L, Chen Y, Zheng Y M, Bai H, Jiang L. Controlling water capture of bioinspired fibers with hump structures. Advanced Materials, 2011, 23, 5486.

[15] Katsoulas N, Bartzanas T, Kitta,E. Effects of anti-drip polyethylene covering films on microclimate and crop production. Acta Horticulturae, 2012, 952, 209-215.

[16] Wu D, Wu S Z, Chen Q D, Zhang Y L, Yao J, Yao X, Niu L G, Wang J N, Jiang L, Sun H B. Curvature-driven reversible in situ switching between pinned and roll-down superhydrophobic states for water droplet transportation. Advanced Materials, 2011, 23, 545.

[17] Yunker P J, Still T, Lohr M A, Yodh A G. Suppression of the coffee-ring effect by shape-dependent capillary interactions. Nature, 2011, 476, 308-311.

[18] Allen Y Y, Wu L, Dave F F, James L L. Overview of polymer micro/nanomanufacturing for biomedical applications. Advances in Polymer Technology, 2008, 27, 188-198.

[19] Marina A G L, Rémy T, Yves L, Jan-Anders E M, Caroline C, Aïcha $\mathrm{H}$ W, Philippe C, Yvan Z, Diego F. UV-nanoimprint lithography and large area roll-to-roll texturization with hyperbranched polymer nanocomposites for light-trapping applications. Solar Energy Materials and Solar Cells, 2012,103, 147-156.

[20] Hu X L, Liu S, Zhou G Y, Huang Y B, Xie Z G, Jing X B. Electrospinning of polymeric nanofibers for drug delivery applications. Journal of Controlled Release, 2014, 185, 12-21.

[21] Yin Z F, Sun L, Zou H L, Cheng E. Two dimensional PMMA nanofluidic device fabricated by hot embossing and oxygen plasma assisted thermal bonding methods. Nanotechnology, 2015, 26, 215302

[22] Heidi O, Diane D C, Michael V, Jürgen V E, Manly C, Pieter W, Wim D M, Hugo T. Dual fiber optical trapping in a polymer-based microfluidic chip. Proceedings of SPIE, Brussels, Belgium, 2016, 9888, 1-9

[23] Toosi S F, Moradi S, Ebrahimi M, Hatzikiriakos S G. Microfabrication of polymeric surfaces with extreme wettability using hot embossing. Applied Surface Science, 2016, 378, 426-434.

[24] Moradi S, Kamal S, Englezos P, Hatzikiriakos S G. Femtosecond laser irradiation of metallic surfaces: Effects 
Li et al.: Facial Fabrication of Biomimetic Water Pinning Microstructures on Polyethylene Surfaces with Robust Superhydrophobic Wetting State

of laser parameters on superhydrophobicity. Nanotechnology, 2013, 24, 415302.

[25] Dongwhi C, Donghyeon Y, Dong S K. One-step fabrication of transparent and flexible nanotopographical-triboelectric nanogenerators via thermal nanoimprinting of thermoplastic fluoropolymers. Advanced Materials, 2015, 27, 7386-7394.

[26] Shin H K, Min G K, Jeong J K, Pyeong A L, Bo H K, Young H C. Micro/nano-hybrid lens for enhancing light extraction using micro-milling and anodic aluminium oxide (AAO). Journal of Micromechanics and Microengineering, 2016, 26, 015010.

[27] Zhou L, Dong X X, Zhou Y, Su W M, Chen X L, Zhu Y F, Shen S. Multiscale micro-Nano nested atructures: Engineered surface morphology for efficient light escaping in organic light-emitting diodes. ACS Applied Materials Interfaces, 2015, 7, 26989-26998.

[28] Chen P L, Hong R H, Yang S Y. Hot-rolled embossing of microlens arrays with antireflective nanostructures on optical glass. Journal of Micromechanics \& Microengineering, 2015, 25, 095001

[29] Heckele M, Bacher W, Müller K D. Hot embossing-the molding technique for plastic microstructures. Microsystem Technologies, 1998, 4, 122-124.

[30] Vasiliy N G, Hsieh Y C, Petzold N O, Faris R A, Yuen P K. Hot embossing of plastic microfluidic devices using poly(dimethylsiloxane) molds. Journal of Micromechanics \& Microengineering, 2011, 21, 17002-17009.

[31] Lee K L, Wu T Y, Hsu H Y, Yang S Y, Wei P K. Low-cost and rapid fabrication of metallic nanostructures for sensitive biosensors using hot-embossing and dielectric-heating nanoimprint methods. Sensors, 2017, 17, 1548.

[32] Wenzel R N. Resistance of solid surfaces to wetting by water. Industrial \& Engineering Chemistry, 1936, 28, 988-994.

[33] Kwok D Y, Neumann A W. Contact angle measurement and contact angle interpretation. Advances in Colloid and Interface Science, 1999, 81, 167-249.

[34] Lum K, David C, Weeks J D. Hydrophobicity at small and large length scales. Journal of Physical Chemistry B, 1999,
103, 4570-4577.

[35] Gennes P G, Brochard-Wyart F, Quéré D. Capillarity and wetting phenomena-drops, bubbles, pearls, waves. Physics Today, 2004, 57, 66-67.

[36] Furmidge C G. Studies at phase interfaces. I. the sliding of liquid drops on solid surfaces and a theory for spray retention. Journal of Colloid Science, 1962, 17, 309-324.

[37] Kim H Y, Lee H J, Kang B H. Sliding of liquid drops down an inclined solid surface. Journal of Colloid \& Interface Science, 2002, 247, 372-380.

[38] Lee A, Moon M W, Lim H, Kim W D, Kim H Y. Water harvest via dewing. Langmuir, 2012, 28, 10183-10191.

[39] Hannu T, Mikko T, Jurkka K. Adhesion mechanism of water droplets on hierarchically rough superhydrophobic rose petal surface. Journal of Nanomaterials, 2011, 6, 818707.

[40] Law J B K, Andrew M H N, He A Y, Low H Y. Bioinspired ultrahigh water pinning nanostructures. Langmuir, 2014, 30, 325-331.

[41] McLauchlin M, Yang D, Aella P, Garcia A A, Picraux S T, Hayes M A. Evaporative properties and pinning strength of laser-ablated, hydrophilic sites on lotus-leaf-like, nanostructured surfaces. Langmuir, 2007, 23, 4871-4877.

[42] Lai Y K, Gao X F, Zhuang H F, Huang J Y, Lin C J, Jiang L. Designing superhydrophobic porous nanostructures with tunable water adhesion. Advanced Materials, 2009, 21, 3799-3803.

[43] Sanjay S L, Sunetra L D, Charles K, Hiroaki I, Ganesan V, Venkateswara R A, Pratap B W, Satish C G. Sliding behavior of water drops on sol-gel derived hydrophobic silica films. Applied Surface Science, 2010, 256, 3259-3264.

[44] Liu M J, Zheng Y M, Zhai J, Jiang L. Bioinspired super-antiwetting interfaces with special liquid-solid adhesion. Accounts of Chemical Research, 2010, 43, 368-377.

[45] Zhu H, Guo Z G, Liu W M. Adhesion behaviors on superhydrophobic surfaces. Chemical Communications, 2014, 50, 3900-3913. 